\title{
Choriocarcinomas May Be Presented with Abnormal Manifestations
}

\author{
Alpaslan Tanoglu, ${ }^{1,}$ and Tolga Duzenli ${ }^{1}$ \\ ${ }^{1}$ GATA Haydarpasa Training Hospital Department of Gastroenterology, 34668, Uskudar, Istanbul, Turkey \\ "Corresponding author: Alpaslan Tanoglu, MD, Gastroenterologist, GATA Haydarpasa Training Hospital Department of Gastroenterology, 34668, Uskudar, Istanbul, Turkey. Tel: \\ +90-2165422020, Fax: +90-2165423504, E-mail: alpaslantanoglu@yahoo.com
}

Received 2017 August 25; Accepted 2017 September 02.

Keywords: Choriocarcinoma, Manifestation

\section{Dear Editor,}

We read, with great interest, the article by Zohreh Yousefi et al. (1), who reported abnormal presentations of choriocarcinoma and review of the literature. The aim of the presentation was the management and outcome of a case series of choriocarcinoma patients with abnormal manifestation. They have presented 4 patients with the initial manifestation as septic shock, severe gastrointestinal hemorrhage, postpartum infection, and postmenopausal bleeding. With accurate diagnosis and appropriate treatment, 3 of 4 patients have cured and, now, set out free of disease. This case series points out important issues about the initial manifestations of choriocarcinomas.

First of all, choriocarcinomas are the most aggressive and rapidly arising germ cell tumors. It generally arises as gestational choriocarcinoma from fetal trophoblasts of a previous hydatidiform mole pregnancy. It develops infrequently from germ cells in testis or ovary. In males, it commonly bears as a component of testicular mixed germ cell tumors, whereas its pure form portrays less than 3\% of all cases (2). Metastasis is most common to lung, liver, and brain, and a majority of patients already have had metastasis to multiple sites at the time of diagnosis (3). Nevertheless, the metastasis of stomach that causes massive gastrointestinal hemorrhage is extremely rare; yet, it may represent a life-threatening emergency as in the case we presented a few years ago (4). Furthermore, germ cell cancers with non-pulmonary metastases have poor prognosis and the management of these patients requires a rapid and multimodal approach. Secondly, with new diagnostic techniques and facilities, metastatic choriocarcinomas may have much more opportunities of earlier diagnosis. In addition, there are significant progresses over the past decades in chemotherapy management and despite metastatic disease, this disease could be considered curable.
In conclusion, the possibility of choriocarcinoma should be kept in mind for unusual presentations, such as gastrointestinal hemorrhages secondary to metastases, rather than the primary tumor, which may go unnoticed.

\section{References}

1. Yousefi Z, Mottaghi M, Rezaei A, Ghasemian S. Abnormal Presentation of Choriocarcinoma and Literature Review. Iran J Cancer Prev. 2016;9(2):4389. doi: 10.17795/ijcp-4389. [PubMed: 27482332].

2. Shimizu S, Nagata Y, Han-yaku H. Metastatic testicular choriocarcinoma of the skin. Report and review of the literature. Am J Dermatopathol. 1996;18(6):633-6. [PubMed: 8989940].

3. Lee SC, Kim KH, Kim SH, Lee NS, Park HS, Won JH. Mixed testicular germ cell tumor presenting as metastatic pure choriocarcinoma involving multiple lung metastases that was effectively treated with high-dose chemotherapy. Cancer Res Treat. 2009;41(4):229-32. doi: 10.4143/crt.2009.41.4.229. [PubMed: 20057969].

4. Yazgan Y, Oncu K, Kaplan M, Tanoglu A, Kucuk I, Ozari HO, et al. Upper gastrointestinal bleeding as an initial manifestation of metastasis secondary to choriocarcinoma. Turk J Gastroenterol. 2013;24(6):565-7. [PubMed: 24623301]. 